\title{
Distribución geográfica y ecoepidemiología de la fauna de triatominos (Reduviidae: Triatominae) en la Isla Margarita del departamento de Bolívar, Colombia
}

\author{
Omar Cantillo-Barraza', Andrés Gómez-Palacio', Diego Salazar², Ana María Mejía-Jaramillo, \\ Jaime Calle ${ }^{2}$, Omar Triana ${ }^{1}$ \\ 1 Grupo de Biología y Control de Enfermedades Infecciosas, BCEI, Sede de Investigación Universitaria (SIU), \\ Instituto de Biología, Universidad de Antioquia, Medellín, Colombia \\ 2 Grupo de Ecoepidemiología y Control Biológico, Instituto de Biología, Universidad de Antioquia, Medellín, \\ Colombia
}

Introducción. Los reportes sobre la diversidad de triatominos y algunos aspectos ecoepidemiológicos en la Isla Margarita se han limitado sólo a dos de los cinco municipios que la conforman. El conocimiento de las especies, su hábitat y la infección, es fundamental para establecer el riesgo en áreas endémicas para la enfermedad de Chagas.

Objetivo. Describir la fauna de triatominos, la distribución y la infección por Trypanosoma cruzi en la Isla Margarita, con el fin de aportar conocimientos que permitan establecer y estratificar el riesgo de transmisión de la zona.

Materiales y métodos. Se realizó la captura de triatominos en las viviendas y el extradomicilio. En las viviendas se hizo búsqueda activa en el intradomicilio y el peridomicilio, mientras en el extradomicilio, se hizo en palmas y nidos de aves. La infección por T. cruzi se determinó por PCR a partir de las heces.

Resultados. Se capturaron 1.154 insectos de cinco especies en los municipios de la isla, Triatoma maculata y Rhodnius pallescens mostraron altos índices de infección en el intra domicilio y en el peridomicilio en Mompós y Talaigua Nuevo. En las palmas, $R$. pallescens fue hallado infectado en todos los municipios y Eratyrus cuspidatus sólo en San Fernando y Margarita.

Conclusión. La presencia de triatominos infectados en las viviendas y en las palmas en los cinco municipios de la isla, llama la atención sobre el potencial riesgo que representan estos insectos en la zona.

Palabras clave: enfermedad de Chagas, Triatominae, Trypanosoma cruzi, Triatoma spp., Rhodnius spp., Colombia.

Distribution and ecoepidemiology of the triatomine fauna (Hemiptera: Reduviidae) in Margarita Island, Bolívar, Colombia

Introduction. Information concerning to triatomine diversity and some eco-epidemiologic aspects on Margarita Island has been recorded only from two of the five counties on the island. Knowledge about species habitat and their natural infection is essential to establish the risk for Chagas disease in endemic areas.

Objective. The distribution of triatomine insect fauna and its infection with Trypanosoma cruzi was described in order to establish and to stratify the risk of Chagas disease transmission.

Material and methods. Each of the 5 counties on Margarita Island were surveyed for triatomid insects inside and outside each dwelling. In the extradomicilary area, searches were conducted in the palms and bird nests located within forests and in pastures near domiciles. Infection with T. cruzi was determined amplifying by PCR the DNA extracted from triatomine feces.

Results. Five species of Reduviidae were recovered among the 1,154 triatomines captured in the 5 counties. Triatoma maculata and Rhodnius pallescens showed high infection rates within dwellings and as well as in the peridomestic areas in Mompós and Talaigua Nuevo. On the palm trees, only $R$. pallescens and Eratyrus cuspidatus were found infected, and only in San Fernando and Margarita. In Cicuco, only $R$. pallescens was infected. Presence of Triatoma dimidiata was also ascertained. Conclusion. Infected triatomines were present in houses and on palm trees in all counties on the island. These observations indicate a potential risk of Chagas across the entire island; furthermore the presence of $T$. dimidiata, a very efficient Chagas vector, emphasizes the need to establish its epidemiological status on the island.

Key words: Chagas' disease, Triatominae, Trypanosoma cruzi, Triatoma spp., Rhodnius spp., Colombia. 
Actualmente, cerca de 7'694.500 de personas en 21 países de Norteamérica, Centroamérica y Suramérica, se encuentran infectadas con Trypanosoma cruzi, agente etiológico de la enfermedad de Chagas (1). T. cruzi es transmitido principalmente por insectos hematófagos de la subfamilia Triatominae, en la cual se cuentan actualmente 141 especies (2,3). Aunque no todas estas especies tienen importancia en la epidemiología de la enfermedad, sí pueden considerarse como vectores potenciales. Entre las especies más reconocidas como vectores, se encuentran Rhodnius prolixus, $R$. pallescens, Triatoma infestans, T. dimidiata, T. maculata, $T$. brasiliensis, $T$. sordida y Panstrongylus megistus, dado que son consideradas las responsables de más de $80 \%$ de la transmisión en las áreas endémicas (4).

En Colombia, actualmente se han descrito 26 especies, de las cuales, 15 se han encontrado con infecciones naturales por T. cruzi (5). De este grupo se destacan $R$. prolixus y $T$. dimidiata como las de mayor importancia epidemiológica, principalmente por su presencia intradomiciliaria (6). No obstante, la presentación de brotes de la enfermedad en sitios donde no se registran poblaciones intradomiciliarias de estos vectores, hace pensar en la intervención de vectores menos reconocidos, como Eratyrus cuspidatus (7), Panstrongylus geniculatus (8), T. maculata y $R$. pallescens (9), entre otros, especies de las cuales se desconoce muchos aspectos de su ecoepidemiología. Por tal razón, el conocimiento de las características biológicas de los vectores que están presentes en las zonas con transmisión activa y el riesgo que pueden representar para la población residente, es fundamental en la elección de medidas que sirvan de complemento a las estrategias tradicionales de control y vigilancia vectorial (4).

En la Isla Margarita del departamento de Bolívar, el conocimiento de los triatominos presentes en las viviendas, así como el nivel de infección con $T$. cruzi, es limitado, dado que sólo se tienen algunas aproximaciones derivadas del Programa Nacional de Prevención y Control de la Enfermedad de

Correspondencia:

Omar Cantillo-Barraza, Grupo de Biología y Control de Enfermedades Infecciosas, Sede de Investigación Universitaria, Universidad de Antioquia, Calle 62 №.52-59, laboratorio 620, Medellín, Colombia.

Fax: (574) 2196520

omarcantillo@gmail.com

Recibido:19/11/09; aceptado:20/04/10
Chagas y la Cardiopatía Infantil en su nodo occidental realizado en 1998, cuando se registró la presencia de pocos insectos, sin infección por $T$. cruzi, en las viviendas (Restrepo M, Restrepo BN, Parra GJ, Salazar CL. Estudio sobre tripanosomiasis americana en el Instituto Colombiano de Medicina Tropical Medellín, Colombia. Programa Nacional de Prevención y Control de la enfermedad de Chagas en los departamentos de Antioquia, Bolívar, Córdoba, Magdalena y Sucre. En: Guhl F, Jaramillo CA, editores. Curso taller internacional "Sistemas de información geográfica, sensores remotos y genética poblacional de vectores y parásitos aplicados al control de la enfermedad de Chagas. Santafé de Bogotá. 2002. p. 117).

Sin embargo, la presentación de casos de infección que conllevaron al fallecimiento de un menor de 9 años en la cabecera de Mompós en 2003 y la detección de dos menores de cinco años seropositivos en el municipio de Talaigua Nuevo en el mismo año, sugiere una posible transmisión activa en la zona (9) (Cortés L. Triatominos (Reduviidae: Triatominae) en (Mompox Bolívar, Colombia). En: Resúmenes, XXXIII Congreso de Entomología. SOCOLEN. Sociedad Colombiana de Entomología. Manizales. 2006. p. 100).

Este panorama fue confirmado por la presencia de $T$. maculata, E. cuspidatus, P. geniculatus y $R$. prolixus en $20 \%$ de las viviendas del municipio de Talaigua Nuevo, con niveles de infección por flagelados en heces de 58,33\% (9, Cortés L. Triatominos (Reduviidae: Triatominae) en (Mompox, Bolívar, Colombia). En: Resúmenes, XXXIII Congreso de Entomología. SOCOLEN. Sociedad Colombiana de Entomología. Manizales. 2006. p.100). Estos estudios indican que en la isla existen especies con potencial riesgo epidemiológico y ponen de manifiesto la necesidad de establecer estos aspectos en los demás municipios.

Por todo lo anterior, este trabajo pretendió actualizar la distribución, los hábitats y el estado de infección natural con T. cruzi de los triatominos, con el fin de establecer y estratificar el riesgo de transmisión en los municipios que conforman la Isla Margarita del departamento de Bolívar.

\section{Materiales y métodos}

\section{Zona de estudio}

El estudio fue realizado en la cabecera y en tres corregimientos del municipio de Mompós, y en las cabeceras de los municipios de San Fernando, Talaigua Nuevo, Cicuco y Margarita. Estos se 
encuentran ubicados en la Isla Margarita (Isla de Mompós) del departamento de Bolívar, Colombia (figura 1). Debido al tamaño y a las características urbanísticas del municipio de Mompós, se dividió en zona urbana (conformada por la cabecera municipal) y zona rural (conformada por sus tres principales corregimientos: La Rinconada, Tierrafirme y Guataca).

La selección de las viviendas para cada municipio se hizo por asignación aleatoria de las manzanas y de las casas. Para el cálculo de la muestra se tuvieron en cuenta los siguientes parámetros: universo 17.166 viviendas (10); infestación esperada, 50\%; error de muestreo, 4\% (11). El tamaño de la muestra fue de 580 casas, de las cuales, se revisó el 96,5\% (560 viviendas), distribuidas proporcionalmente según el número total de casas de cada municipio, de la siguiente manera: Mompós urbano, 130 (22,4\%); Mompós rural, 140 (24,1\%); Talaigua Nuevo, 74 (12,8\%); Cicuco, 72 (12,5\%); San Fernando, 72 (12,5\%), y Margarita, 72 (12,5\%). En la elección de las palmas de la especie Attalea butyracea, se tuvo en cuenta la cercanía a las viviendas evaluadas en cada municipio.

\section{Búsqueda de triatominos}

Se realizaron cuatro muestreos entre los años 2006 y 2008 , en los cuales se hizo búsqueda activa de triatominos en las viviendas y el extradomicilio. Cada una de las viviendas se dividió en intradomicilio y peridomicilio, el cual fue definido para este estudio como la parte trasera perteneciente a la viviendas delimitadas por cercas, donde se encuentran estructuras construidas por los propietarios (patios), y se realizó una búsqueda exhaustiva mediante la metodología hombre-hora (12), empleando 15 minutos para cada ambiente.

En el interior de las viviendas se revisaron los muebles y los enseres presentes en las habitaciones, salas, comedores, cocinas y ranchos anexos; en los patios se revisaron estructuras como gallineros, palomares, chiqueros, ranchos, dormitorio de los perros, cúmulos de madera o ladrillos.

Una vez finalizada la búsqueda, al jefe de familia se le entregó un recipiente plástico para el almacenamiento de los triatominos que lograran capturar las personas que habitaban la vivienda.

Para los triatominos presentes en los domicilios, se calcularon los índices convencionales de acuerdo con la definición propuesta por la Organización
Mundial de la Salud (OMS) (1991): índice de infestación (II V), índice de densidad, (ID), índice de infección (IIN), e índice de colonización (IC) aplicado a las viviendas como unidades.

Para la búsqueda de triatominos en el extradomicilio, se disecaron 50 palmas de la especie $A$. butyracea, localizadas en potreros de las fincas que rodean las poblaciones o en lotes dentro de las mismas.

Para la elección de las palmas se tuvieron en cuenta aquellos especímenes adultos con estípites con más de tres metros de altura, bajo los siguientes criterios: palmas ubicadas a una distancia menor de un kilómetro de las casas, palmas taladas por sus propietarios que no tuvieran más de 15 días de haber sido cortadas, y palmas cuyos propietarios manifestaron su intención de talarlas.

La recolección de triatominos en las palmas se llevó a cabo mediante la metodología previamente descrita (13), con revisión minuciosa de todas las brácteas de la palma, comenzando desde las más viejas ubicadas en la zona inferior, hasta llegar a las más altas o jóvenes. La ubicación geográfica de la palma fue georreferenciada con un GPS (Global Positioning System), marca Magellan®.

\section{Clasificación y evaluación parasitológica de triatominos}

Los insectos capturados se identificaron siguiendo las claves taxonómicas de referencia para triatominos (14) y se clasificaron según su estadio de desarrollo.

La determinación de la infección por $T$. cruzi en los insectos recolectados, se hizo por observación directa de las heces al microscopio y posterior confirmación por reacción en cadena de la polimerasa (PCR). De los 1.111 ejemplares capturados en el extradomicilio, se evaluaron por observación directa 98 individuos (13 del estadio IV, 27 del V y 58 adultos), de los cuales, 26 se confirmaron por PCR.

Todos los insectos recolectados en las casas se analizaron por ambas técnicas. Los insectos negativos para flagelados en heces, fueron disecados y su contenido intestinal almacenado en PBS estéril a $4^{\circ} \mathrm{C}$ hasta la realización de la PCR para descartar la presencia de $T$. cruzi. Para la confirmación de la infección con $T$. cruzi, se extrajo ADN a partir de las heces o el contenido intestinal por el método de salting out $(15,16)$ y posterior amplificación por PCR del ADN satélite de $T$. cruzi según lo reportado (17). 
Para esta reacción se utilizaron los iniciadores TCZF (5'-GCTCTTGCCCACA(AC)GGGTGC-3') y TCZR (5'-CCAAGCAGCGGATAGTTCAGG-3'), los cuales amplifican un fragmento de $188 \mathrm{pb}$ especifico para T. cruzi (17). La PCR se llevó a cabo en un volumen final de $50 \mu$ de reacción que contenía $5 \mu \mathrm{l}$ de $\mathrm{ADN}$ de heces, $3 \mu \mathrm{l}$ de $\mathrm{MgCl}_{2} 3$ $\mathrm{mM}, 5 \mu \mathrm{l}$ de solución tampón $1 \mathrm{X}, 2 \mathrm{pmol} / \mu \mathrm{l}$ de cada iniciador, 0,5 $\mu$ le dNTP a 0,25mM, 1,5 unidades de Taq ADN polimerasa platinum (Invitrogen ${ }^{\circledR}$ ) y $32,2 \mu l$ de agua bidestilada ultrapura.

La amplificación se hizo en un termociclador PTC100 a una temperatura inicial de $94^{\circ} \mathrm{C}$ por 3 minutos, seguida de 40 ciclos de $94^{\circ} \mathrm{C}$ por 45 segundos, $68^{\circ} \mathrm{C}$ por un minuto y $72^{\circ} \mathrm{C}$ por 1 minuto, y un ciclo final de $72^{\circ} \mathrm{C}$ por 10 minutos. Los productos amplificados se analizaron por electroforesis en gel de agarosa al 1,5\% teñido con bromuro de etidio y se visualizaron bajo luz ultravioleta.

\section{Resultados}

Triatominos recolectados en los domicilios e infección con T. cruzi

El índice de infestación en los cinco municipios fue de $5 \%$ (28/560). Se recolectaron 43 triatominos, 41 de la especie T. maculata $(95,3 \%)$ y $2(4,7 \%)$ de $R$. pallescens distribuidos en los municipios de Mompós y Talaigua Nuevo. En los municipios de San Fernando, Margarita y Cicuco no se hallaron insectos en las viviendas, mientras $T$. maculata fue encontrado en el intradomicilio y peridomicilio. $R$. pallescens se encontró sólo en el interior de las viviendas en Talaigua Nuevo y en el corregimiento de La Rinconada de Mompós. En el cuadro 1 se detallan los índices entomológicos estimados para T. maculata en cada municipio.

Cinco ninfas de $T$. maculata fueron capturadas en patios asociados a gallineros, mientras los restantes 36 adultos fueron hallados tanto en el intradomicilio como en el peridomicilio (cuadro 1). T. maculata presentó altos niveles de infección con $T$. cruzi en Talaigua Nuevo de $71,4 \%$, en Tierrafirme de $64,7 \%$ y en La Rinconada del $50 \%$. Los dos especímenes de $R$. pallescens capturados fueron especímenes adultos, ambos positivos para $T$. cruzi.

\section{Triatominos en el extradomicilio e infección con T. cruzi}

Se capturaron 1.111 triatominos en las palmas evaluadas en todos los municipios excepto en Mompós urbano (figura 1), de los cuales, la gran mayoría (1.000 ejemplares) se encontraron en los estadios juveniles I, II y III. El 96\% correspondió a R. pallescens (1066/1.111), el 3,8\% a E. cuspidatus (42/1.111), el 0,2\% a $T$. dimidiata (2/1.111) y el $0,09 \%$ a C. pilosa (1/1.111). R. pallescens fue la única especie capturada en todos los sitios de muestreo, mientras que $E$. cuspidatus se encontró en los municipios de Talaigua Nuevo, Margarita y San Fernando, T. dimidiata, en Margarita, y $C$. pilosa, en San Fernando. El número de individuos fluctuó entre 0 y 129 insectos por palma, con una tasa de infestación global de $82 \%$ y una media de 22,22 insectos por palma.

Del total de 111 triatominos de los estadios IV y V, y adultos, 98 fueron evaluados para infección por flagelados en todos los sitios de muestreo y mostraron rangos de infección de $23,8 \%$ en

Cuadro 1. Indicadores entomológicos para Triatoma maculata estimados en cinco municipios y tres corregimientos de Mompós en la Isla Margarita del departamento de Bolívar. Se muestra el número de individuos recolectados en cada sitio.

\begin{tabular}{|c|c|c|c|c|c|c|c|c|}
\hline \multirow{3}{*}{$\begin{array}{l}\text { Índices } \\
\text { entomológicos } \\
\text { para T. maculata } \\
\text { (porcentaje) }\end{array}$} & \multirow{3}{*}{ Urbano } & \multicolumn{3}{|c|}{ Mompós } & \multirow[b]{3}{*}{$\begin{array}{c}\text { Talaigua } \\
\text { Nuevo }\end{array}$} & \multirow[b]{3}{*}{ San Fernando } & \multirow[b]{3}{*}{ Margarita } & \multirow[b]{3}{*}{ Cicuco } \\
\hline & & \multicolumn{3}{|c|}{ Rural } & & & & \\
\hline & & Tierrafirme & La Rinconada & Guataca & & & & \\
\hline $\mathrm{n}$ & 2 & 21 & 3 & 0 & 15 & 0 & 0 & 0 \\
\hline IIVD & 0 & 12,8 & 2,8 & 0 & 6,8 & 0 & 0 & 0 \\
\hline IIVPD & 1,5 & 17,1 & 1,4 & 0 & 13,5 & 0 & 0 & 0 \\
\hline IIC & 0 & 0 & 0 & 0 & 0 & 0 & 0 & 0 \\
\hline TD & 0,015 & 0,3 & 0,04 & 0 & 0,2 & 0 & 0 & 0 \\
\hline IIN & 0 & 64,7 & 50 & 0 & 71,4 & 0 & 0 & 0 \\
\hline ID & & & & 57,1 & & & & \\
\hline
\end{tabular}

(n); el IIVD, índice de infestación domiciliaria; IIVPD, índice de infestación peridomiciliaria; IIC, índice de colonización; TD, tasa de densidad; IIN, índice de infección natural por T. cruzi; y el ID, índice de dispersión. 


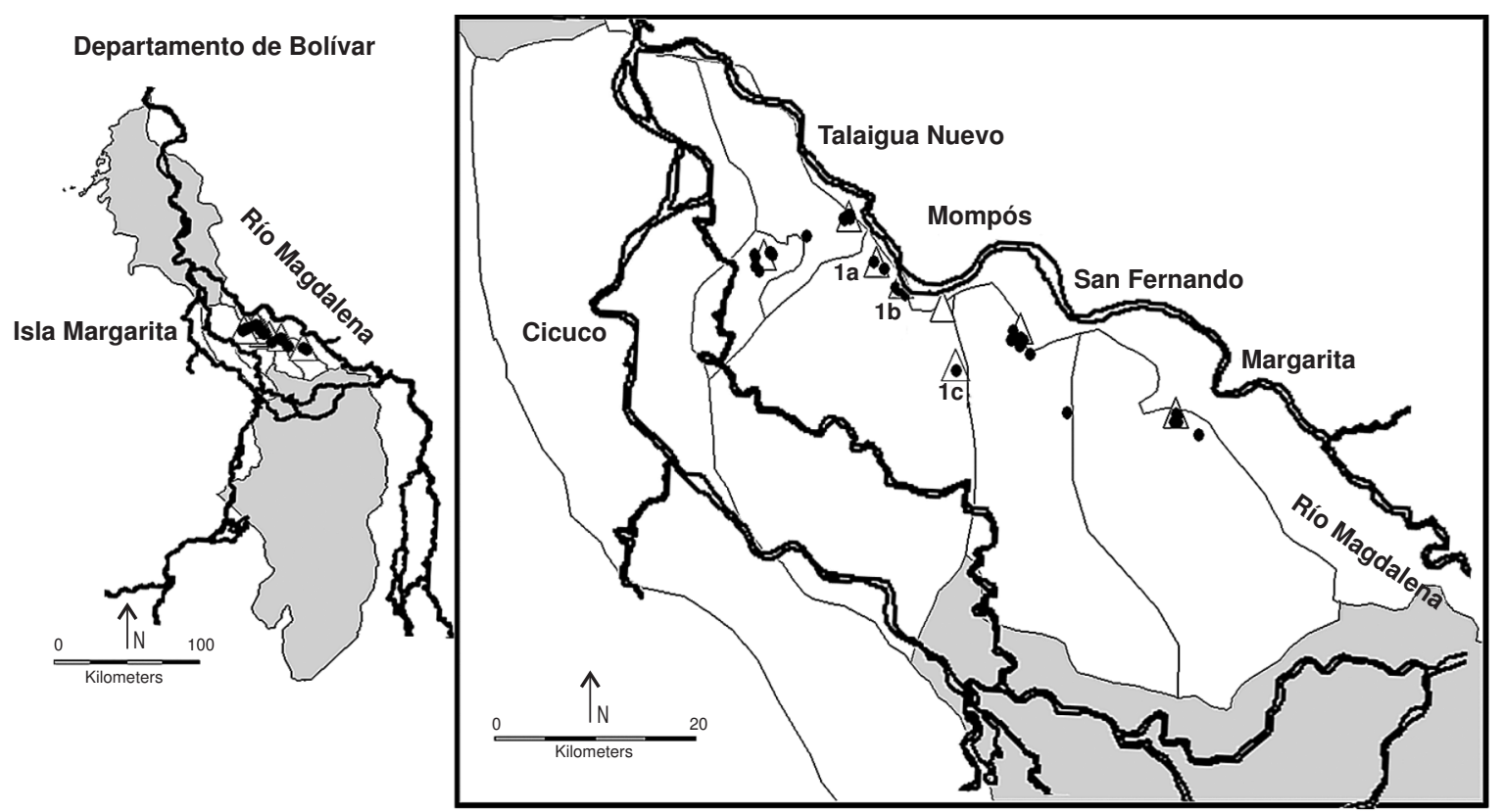

Figura 1. Mapa del departamento de Bolívar donde se indican los municipios que conforman la Isla Margarita en los que se hizo la búsqueda activa de triatominos. Igualmente, se muestran los tres corregimientos del municipio de Mompós incluidos: La Rinconada (1a), Tierrafirme (1b) y Guataca (1c). Los triángulos indican la ubicación de las cabeceras municipales y de los corregimientos estudiados, y los círculos indican la ubicación de las palmas $A$. butyracea con presencia de triatominos infectados naturalmente con T. cruzi.

Tierrafirme y de $100 \%$ en Guataca (Mompós rural). Para los municipios de Talaigua Nuevo, San Fernando y Margarita, se destaca el elevado número de insectos recolectados y un importante porcentaje de infección por flagelados, $72,2 \%, 80 \%$ y $73,6 \%$, respectivamente.

De las 26 muestras seleccionadas de todos los sitios de captura para la confirmación por PCR de la infección con $T$. cruzi, sólo 3 resultaron negativas, lo cual muestra la alta prevalencia de este parásito en la zona.

\section{Discusión}

Las estrategias de control y vigilancia vectorial para la enfermedad de Chagas se fundamentan en el conocimiento de las características biológicas y ecoepidemiológicas de los vectores responsables de la transmisión de $T$. cruzi $(1,3,18)$.

De las cinco especies halladas en la isla Margarita, sólo $C$. pilosa es considerada sin ningún riesgo epidemiológico $(4,7,18$, Angulo VM, Tarazona Z, Reyes A, Gutiérrez R, Sandoval CM. Programa nacional de prevención y control de la enfermedad de Chagas y la cardiopatía infantil; nodo nororiental. En: Angulo VM, editor. Taller internacional sobre control y manejo de la tripanosomiasis americana. Bucaramanga; 1999. p. 99-108.).
T. maculata se encontró en los domicilios de Talaigua Nuevo, Mompós urbano y en dos corregimientos de la zona rural de Mompós (La Rinconada y Tierrafirme). Solamente individuos adultos se encontraron simultáneamente en el intradomicilio y el peridomicilio $(\mathrm{IC}=0)$, lo que indica el posible movimiento activo de estos entre ambos ecótopos. La presencia de T. maculata en los peridomicilios (IIVP de 17,1\% para Tierrafirme y de $13,5 \%$ para Talaigua Nuevo) y su asociación fundamentalmente con gallineros, contrastan con los altos niveles de infección natural encontrados (IIN entre $50 \%$ y $71 \%$ ).

Este hecho llama la atención sobre las fuentes alimenticias de estos insectos y su posible papel en la presentación de los focos de infección reportados en esta zona. T. maculata es una de las especies con mayor distribución en Colombia. Sin embargo, su incriminación directa como vector a humanos no se ha establecido aún. A pesar de que esta especie presenta un elevado nivel de asociación con los domicilios, se han reportado mínimos niveles de infección natural por $T$. cruzi. En este sentido, nuestros resultados llaman la atención sobre esta especie en el país y se sugieren más estudios ecoepidemiológicos que permitan definir su real papel vectorial. 
Aunque en este trabajo no se realizó una encuesta sobre el conocimiento del vector, durante el desarrollo de los muestreos fue reiterativo que los habitantes de los tres municipios donde no se registró la presencia de $T$. maculata, relataran conocer este insecto y haberlo visto en sus casas. De acuerdo con lo anterior, se recomienda realizar una encuesta para evaluar el conocimiento del vector, así como nuevos muestreos dirigidos a barrios con posible presencia del vector, para tener un panorama más claro de la ecoepidemiología de la enfermedad de Chagas en estos tres municipios.

$R$. pallescens fue la otra especie encontrada en las viviendas, lo que debe obedecer a su dispersión desde las palmas, ya que sólo se encontró en estadio adulto. Esta especie se considera un vector secundario por su frecuente "intrusión" en las viviendas (19). En la Isla Margarita, $R$. pallescens está muy bien establecido en $A$. butyracea, con niveles de infestación de $86,9 \%$, como ha sido reseñado en mucha zonas de la región Caribe colombiana (20-23).

En la Isla Margarita, la presencia de este vector se ha reportado en los municipios de Mompós, Talaigua Nuevo y San Fernando $(6,23)$.

En este trabajo se presenta una actualización del estado de infección de este vector para los otros dos municipios y se reporta, por primera vez, la presencia de $R$. pallescens infectado con $T$. cruzi, en los cinco municipios que conforman la isla. $\mathrm{Si}$ bien esta especie está más asociada en nuestro país con el ciclo de transmisión silvestre (19), en Panamá se considera el principal vector de $T$. cruzi debido al contacto habitual con los residentes, producto de las constantes "intrusiones" a las viviendas $(24,25)$.

En los municipios de la Isla de Mompós, la presencia de $R$. pallescens infectado representa un riesgo ocupacional para los trabajadores del campo y para los residentes que se encuentran en cercanías a las palmas, debido a la tendencia de este insecto a realizar visitas esporádicas a las casas $(19,24,25)$, tal como se demostró en el presente trabajo. Para estimar el riesgo que representa esta especie en la zona, se hace necesaria la realización de estudios encaminados a demostrar la influencia de las épocas del año en el aumento del riesgo de transmisión, ya que el incremento de las densidades de ninfas que se presenta en la época seca podría estar relacionado con las "intrusiones" a las casas, condición que explicaría la captura de los individuos de esta especie en las viviendas durante la época seca.

La presencia de E. cuspidatus en la isla de Mompós sólo se había reportado en Talaigua Nuevo y Mompós, sin que se conociera su estado de infección (Cortés L. Triatominos (Reduviidae: Triatominae) en (Mompox Bolívar, Colombia). En: Resúmenes, XXXIII Congreso de Entomología. SOCOLEN. Sociedad Colombiana de Entomología. Manizales. 2006. p. 100).

En este trabajo ampliamos su distribución con infección por T. cruzi a los municipios de San Fernando y Margarita. Esta especie, de hábitos silvestres, ha ido cobrando recientemente gran importancia epidemiológica al ser incriminado como responsable de la transmisión de $T$. cruzi en El Banco, Magdalena, sin indicios de domiciliación (7). E. cuspidatus fue hallado en el ecótopo silvestre compartiendo el hábitat (palmas) con $R$. pallescens, pero con una distribución diferencial por confirmar, con una aparente preferencia por las brácteas caudales, mientras que $R$. pallescens se ubica en las coronas de las palmas. Esta característica ecológica supone que quizá se pueda presentar algún tipo de dominancia zonal entre estas dos especies, que puede tener relación con la capacidad de transporte activo hacia las viviendas.

Aunque esta especie no fue capturada en las viviendas, su presencia en los domicilios fue registrada en Talaigua Nuevo y en el casco urbano de Mompós (9), probablemente, como consecuencia de las visitas realizadas desde las palmas que rodean las poblaciones. Para estas poblaciones silvestres, las comparaciones realizadas en las variaciones de las densidades de población mostraron diferencias estadísticas en el número de individuos recolectados entre la época seca y la de lluvia, lo que podría explicar la presencia en las casas de los individuos reportados en otras investigaciones (Cantillo Omar, datos sin publicar).

T. dimidiata sólo se encontró en el municipio de Margarita, sin infección con $T$. cruzi y compartiendo el hábitat con $R$. pallescens. De este vector ya había reportes en el municipio de San Fernando en las mismas circunstancias (23). La condición silvestre que presenta esta especie en la zona es característica de la llanura del Caribe, donde $T$. dimidiata se presenta en ambiente extradomiciliario asociado a palmeras $(6,20)$, desde donde realizan visitas esporádicas a los domicilios, atraídos por la 
luz (Restrepo M, Restrepo BN, Parra GJ, Salazar CL. Estudio sobre tripanosomiasis americana en el Instituto Colombiano de Medicina Tropical Medellín, Colombia. Programa Nacional de Prevención y Control de La enfermedad de Chagas en los departamentos de Antioquia, Bolívar, Córdoba, Magdalena y Sucre. En: Guhl F, Jaramillo CA, editores. Curso taller internacional "Sistemas de información geográfica, sensores remotos y genética poblacional de vectores y parásitos aplicados al control de la enfermedad de Chagas. Santafé de Bogotá: Universidad de los Andes; 2002. p. 117.), lo cual favorece la aparición de focos de infección. La presencia de este vector en la zona es de gran importancia, ya que actualmente, $T$. dimidiata se considera el vector de $T$. cruzi más importante del continente, luego de las acciones de control sobre $T$. infestans y $R$. prolixus (1).

C. pilosa se recolectó en el municipio de San Fernando; éste es el primer registro de esta especie en este municipio y en el departamento de Bolívar. C. pilosa es un vector silvestre, de poca relevancia ecoepidemiológica, pero reportado con infecciones en Colombia (6). En otros trabajos, esta especie se encontró compartiendo hábitat con $R$. pallescens en una palma colonizada por murciélagos, situación que podría explicar la presencia de sólo una ninfa de esta especie, como consecuencia de una dispersión pasiva (26).

En conclusión, el presente trabajo muestra una situación ecoepidemiológica favorable para la aparición y el establecimiento de focos de transmisión en la Isla Margarita, debido a la presencia de vectores con altos niveles de infección en los alrededores de las poblaciones, la visita esporádica de estos desde las palmas y el establecimiento de T. maculata infectados en los peridomicilios, con tendencia a introducirse en los domicilios.

De acuerdo con lo anterior, se hace necesaria la realización de un estudio ecoepidemiológico que evalúe la real dimensión epidemiológica de la región y, con ello, permita determinar el riesgo de infección que existe para los habitantes de la Isla Margarita.

\section{Conflicto de intereses}

Todos los autores de este trabajo declaramos que no existe conflicto de interés.

\section{Financiación}

Este trabajo se realizó con el apoyo económico de CODI- Universidad de Antioquia, proyecto No. 1245.

\section{Referencias}

1. Guhl F, Lazdins-Held J. Grupo de trabajo científico sobre la enfermedad de Chagas. Geneva: Special Programme for Research and Training in Tropical Diseases; 2007. p. 5-31.

2. Jurberg J, da Silva D, Galvão C. Rhodnius zeledoni sp. nov. afim de Rhodnius paraensis Sherlock, Guitton \& Miles, 1977 (Hemíptera, Reduviidae, Triatominae). Biota Neotrop. 2009;9:123-8.

3. Schofield CJ, Galvão C. Classification, evolution, and species groups within the Triatominae. Acta Trop. 2009;110:88-100

4. Guhl F, Pinto N, Aguilera N. Sylvatic triatominae: A new challenge in vector control transmission. Mem Inst Oswaldo Cruz. 2009;104(Suppl.I):71-5.

5. Sandoval CM, Pabón E, Jurberg J, Galvão C. Belminus ferroae n. sp. from the Colombian north-east, with a key to the species of the genus (Hemiptera: Reduviidae: Triatominae). Zootaxa. 2007;1443:55-64.

6. Guhl F, Aguilera G, Pinto N, Vergara D. Actualización de la distribución geográfica y ecoepidemiología de la fauna de triatominos (Reduviidae: Triatominae) en Colombia. Biomédica. 2007;27(Suppl.1):143-62.

7. Dib J, Barnabe C, Tibayrenc M, Triana O. Incrimination of Eratyrus cuspidatus (Stal) in transmission of Chagas' disease by molecular epidemiology analysis of Trypanosoma cruzi isolates from a geographically restricted area in the north of Colombia. Acta Trop. 2009;3:237-42.

8. Wolf M, Castillo D. Evidencias de domesticación y aspectos biológicos de Panstrongylus geniculatus (Latreille, 1811) (Hemiptera: Reduviidae). Acta Entomol Chil. 2000;24: 77-83.

9. Cortés LA, Suárez H. Triatominos (Reduviidae: Triatominae) en un foco de enfermedad de Chagas en Talaigua Nuevo (Bolívar, Colombia). Biomédica. 2005;25:568-74.

10. DANE. Proyecciones departamentales de población por sexo y edad, Bogotá D.C., 1990-2015 estudios censales N2. Bogotá D.C.: DANE; 2003.

11. Londoño JL. Metodología de la investigación epidemiológica. Tercera edición. Bogotá D.C.: Editorial Manual Moderno; 2004.

12. Pichin R, Fanara DM, Oliveira AM. Comparison of techniques for detection of domestic infestations with Triatoma infestans in Brazil. Trans R Soc Trop Med Hyg. 1981;75:691-4.

13. Romaña C, Pizarro J, Rodas E, Guilbert E. Palm trees as ecological indicators of risk areas for Chagas disease. Trans Roy Soc Trop Med Hyg. 1999;93:1-2.

14. Lent $\mathbf{H}$, Wigodzinsky P. Revision of the Triatominae (Hemiptera, Reduviidae) and their significance as vector of Chagas disease. Bull Am Nat Hist. 1979;163:123-520.

15. Miller SA, Dykes DD, Polesky HF. A simple salting out procedure for extracting DNA from human nucleated cells. Nucleic Acid Res. 1988;16:1215.

16. Sambrook J, Fritsch EF, Maniatis T. In: Molecular cloning: a laboratory manual. Second edition. New York: Cold Spring Harbor Laboratory Press; 1989. 
17. Botero LA, Mejía AM, Triana O. Biological and genetic characterization of two Colombian clones of Trypanosoma cruzi groups I and II. Biomédica. 2007;27(Suppl.1):64-74.

18. Schofield CJ. Global Collaboration for Development of Pesticides for Public Health. Field testing and evaluation of insecticides for indoor residual spraying against domestic vectors of Chagas disease. WHO/CDS/WHOPES/GCDPP. Geneva: World Health Organization; 2001. p. 53.

19. Jaramillo N, Schofield CJ, Gorla D, Caro-Riaño H, Moreno J, Mejía E, et al. The role of Rhodnius pallescens as a vector of Chagas disease in Colombia and Panama. Res Rev Parasitol. 2000;60:75-82.

20. Parra-Henao G, Angulo V, Jaramillo N, Restrepo M. Triatominos (Hemiptera: Reduviidae) de la Sierra Nevada de Santa Marta, Colombia. Aspectos epidemiológicos, entomológicos y de distribución. Revista CES Medicina. 2009;23:17-26

21. Pizarro JC, Romaña C. Variación estacional de una población silvestre de Rhodnius pallescens Barber 1932 (Heteroptera: Triatominae) en la Costa Caribe Colombiana. Bull Inst Fr Etudes Andines.1998;27:309-25.
22. Calle OJ. Vers un controle microbiologique des populations colombiennes de Triatominae, insectes vecteurs de la maladie de Chagas (thèse). Paris: Université de Paris V. René Descartes; 2000.

23. Salazar D, Calle J. Caracterización ecoepidemiológica de Rhodnius pallescens en la Palma Attalea butyracea en la región Momposina (Colombia). Actualidades Biológicas. 2003;78:31-8

24. Vásquez AM, Samudio FE, Saldaña A, Paz HM, Calzada JE. Eco-epidemiological aspects of Trypanosoma cruzi, Trypanosoma rangeliand their vector (Rhodnius pallescens) in Panamá. Rev Inst Med Trop Sao Paulo. 2004;46:217-22.

25. Calzada JE, Pineda VA, Montalvo E, Álvarez D, Santamaría AM, Samudio F, et al. Human trypanosome infection and the presence of intradomicile Rhodnius pallescens in the western border of the Panama canal, Panama. Am J Trop Med Hyg. 2006;74:762-5.

26. Marinkelle $\mathbf{C J}$. The distribution of Colombian triatominae and their infestation with trypanosomatid flagellates. Geneve: World Health Organization; 1968. 